\title{
POLARIZATION IN Be STARS
}

\author{
(Review Paper) \\ GEORGE V. COYNE, S.J. \\ University of Arizona, Tucson, Ariz., U.S.A. \\ and \\ Vatican Observatory, Vatican City State
}

\begin{abstract}
A review of polarization produced in the extended circumstellar disks about Be stars is given. While variability of the polarization and its peculiar wavelength dependence in wide-band continuum measurements are discussed, emphasis is placed upon the recently discovered polarization effects in the emission lines and upon a discussion of models. Scattering in the photosphere of a star, even one rigidly rotating near breakup velocity, does not explain the observations. Better fits to the observations are obtained by scattering from electrons in a flattened gaseous disk extending some 10 stellar radii from the star with electron temperatures $T_{e} \cong 10000 \mathrm{~K}$ and electron densities $N_{e} \cong 10^{12} \mathrm{~cm}^{-3}$. In some stars the emission flux in $\mathrm{H} \alpha$ and $\mathrm{H} \beta$ appears to be partly polarized.
\end{abstract}

\section{Introduction}

One of the ways in which linear polarization can be produced is by the scattering of light from particles which are asymmetrically distributed as seen by the observer. The emission lines observed in the spectra of classical Be stars are said to originate in a flattened gaseous envelope extending some 10 stellar radii out from the photosphere of a rapidly rotating hot star. Thus one might expect to detect linear polarization in these systems as the light from the central star is scattered to the observer from this flattened gaseous disk. One might then employ the polarimetric observations as a means of studying the nature of the extended gaseous envelope about these stars. While we now realize, indeed, that the polarization produced in these envelopes is a powerful means of studying the nature of the envelopes themselves, the original detection of intrinsic polarization in these stars did not come about in such a reasoned, systematic way.

In fact, the first prediction of intrinsic stellar polarization stimulated a search which resulted in the detection of the interstellar polarization. Chandrasekhar (1946) predicted that the linear polarization could be as high as $11 \%$ at the limb of a star with a plane-parallel atmosphere in which there was pure electron scattering. In a search for such polarization in O and B-type stars Hall (1949) and Hiltner (1949) independently discovered the interstellar polarization.

It was about ten years later, as a matter of fact, that the first detection of intrinsic stellar polarization in Be stars was made by Behr (1959) who suspected variable polarization in the shell star, $\gamma$ Cas. On the other hand, the first hint that intrinsic stellar polarization had been observed may be found already in Hall and Mikesell (1950) where, at the end of their catalog, a comment is made about the unexpectedly large polarization in $\zeta$ Tau, considering its color excess.

Intrinsic polarization in stars can be detected in four ways: (1) variability of the polarization; (2) variation of the polarization position angle with wavelength; (3) 
peculiar $p(\lambda)$ for the continuum radiation; (4) changes in the polarization across discrete absorption or emission features. Historically, the first detections of intrinsic polarization were due to observed variations and observational progress was made more or less in the sequence of the four criteria listed above.

Subsequent to Behr's (1959) discovery of variable polarization in $\gamma$ Cas, Shakhovskoi (1962) found variable polarization for $\chi$ Oph and later (Shakhovskoi, 1964) for other Be stars. Still other early-type stars were found to show variable polarization by Vitrichenko and Efimov (1965) and Coyne and Gehrels (1967).

Serkowski (1968) first indicated the peculiar nature of the wavelength dependence of the polarization for stars with extended atmospheres, although a similar $p(\lambda)$ had already been observed for the recurrent nova T Pyxidis by Eggen et al. (1967) and for $\beta$ Lyrae by Appenzeller and Hiltner (1967).

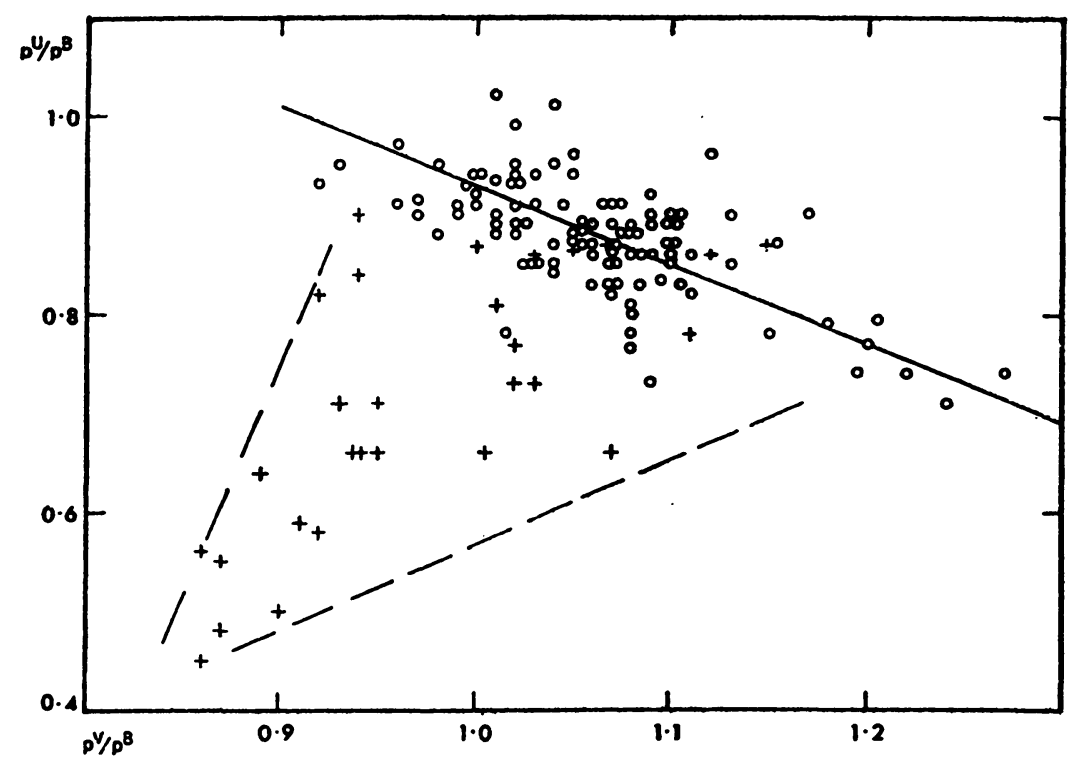

Fig. 1. A plot of polarization ratios for stars which represent the interstellar polarization (open circles) and for Be stars (crosses).

Figure 1 (from Serkowski, 1970) shows a plot of the polarization ratios, ultraviolet-to-blue vs visual-to-blue, for stars which represent the interstellar polarization (open circles) and for emission-line stars (crosses). The emission-line stars have a systematically lower ultraviolet polarization than that for the interstellar medium. Coyne and Kruszewski (1969) also observed this effect and in addition showed that the polarization in the Be stars, as compared to that for the interstellar medium, increased longwards of the Paschen limit. Serkowski (1970) verified this and in Figure 2 his observations of two Southern Be stars p Car (full circles) and $\alpha$ Ara (open circles) are shown contrasted to $p(\lambda)$ curves for the interstellar medium (dashed lines). Here are seen both the decrease in the ultraviolet polarization and the increase beyond the Paschen limit. 


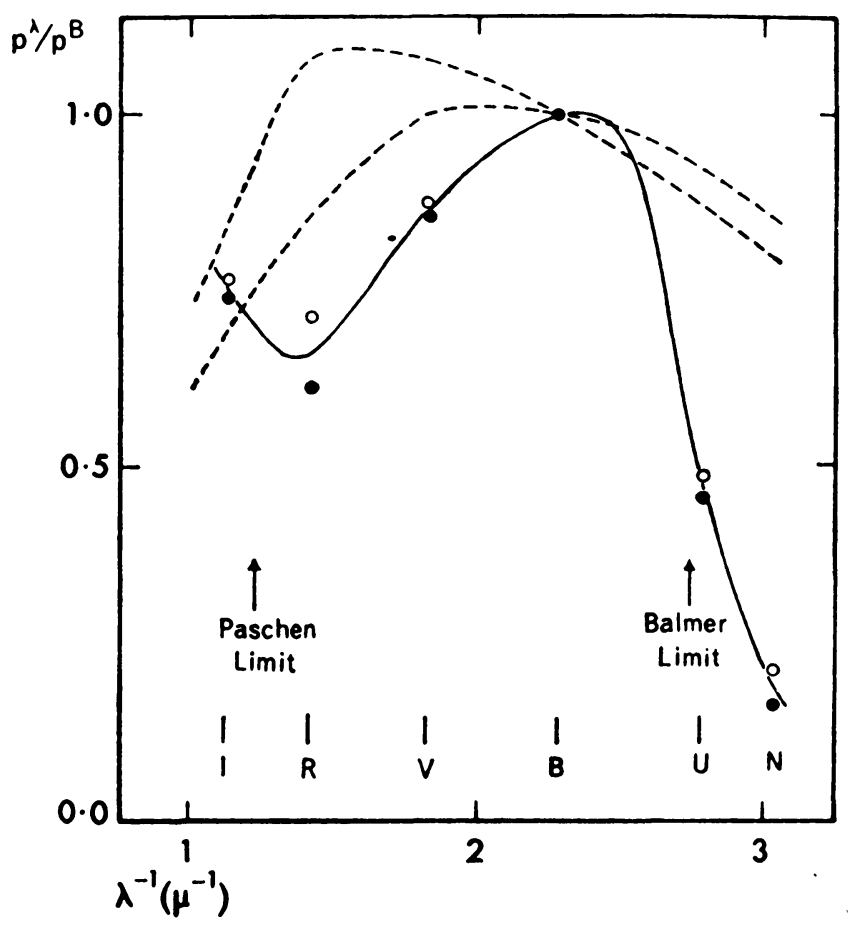

Fig. 2. Polarimetric observations of two Be stars (p Car and $\alpha$ Ara) are shown by solid line; these are compared to two representative curves for the interstellar medium shown by dashed lines.

As we shall see subsequently (Section 2) it is not always easy to separate out the effects of the interstellar and intrinsic polarization. Serkowski (1970) was able to show the diminishing effects of interstellar polarization on a relatively large group of early-type stars which he studied. His results are shown in the polarization-ratio plot of Figure 1 where the apparent apex of the triangular region confining the emissionline stars represents the intrinsic polarization least contaminated by the interstellar polarization. The values for $\alpha$ Ara and p Car, plotted in Figure 2, lie near this apex. Coyne (1971) observed a group of Be stars at high galactic latitudes in seven colors and his $p(\lambda)$ curve together with that of Serkowski can be considered characteristic of the $p(\lambda)$ for the intrinsic polarization in emission-line stars. Indeed, there are differences in the intrinsic $p(\lambda)$ from star to star, but we shall discuss this in Section 2.

To date about $50 \%$ of the $\mathrm{Be}$ and shell stars observed polarimetrically show intrinsic polarization detectable either because the polarization is variable or because the $p(\lambda)$ differs from that for the interstellar medium.

The most recent developments in the polarimetry of Be stars have been observationally, the detection of changes in the polarization across the emission lines and theoretically, the development of more detailed models to explain the observed polarizations. Zellner and Serkowski (1972) report an unpublished observation by Serkowski of a decrease of polarization across the $H \beta$ line of $\zeta$ Tau. Actually Öhman (1934) noted that one wing of $\mathrm{H} \gamma$ in $\beta$ Lyr showed polarization dependent on the phase of the eclipsing binary. Öhman (1946) also predicted that the Chandrasekhar 
effect could cause a variation of as much as $0.8 \%$ polarization across an absorption line Doppler-broadened by rapid stellar rotation. Later Sen and Lee (1961) made a similar prediction. But the first published systematic observations of polarization effects across emission lines were made recently, first by McLean (1974) and Clarke and McLean (1974) and then by several others whose work will be reviewed in Section 4. Observations of polarization effects in spectral lines by Clarke and Grainger (1966), by Derviz (1970) have not been verified (see Hayes and Illing, 1974). The detection of changes in the polarization across the emission lines of some Be stars provides conclusive proof of the presence of intrinsic polarization in these stars and in many cases allows one to isolate the intrinsic from the interstellar polarization and to estimate the electron distribution in the extended envelope. We shall discuss this in Section 4.

As for models to explain the polarimetric observations, it is unlikely that photospheric effects contribute in any significant way. Nagirner (1962) first showed that the polarization of the integrated visible light from the photosphere of a rotationally distorted early-type star does not exceed $0.1 \%$. This was later shown to be true in a more sophisticated analysis by Collins (1970) and by Ruciński (1970). It has been recently shown (Cassinelli and Haisch, 1974; Haisch and Cassinelli, 1976) that the polarization from early-type stars rigidly rotating at breakup velocities does not exceed $0.3 \%$ and that only a highly flattened disk can provide polarization greater than $1.0 \%$. Coyne and Kruszewski (1969) explained the wavelength dependence of the polarization in Be stars by electron scattering in the extended envelope together with hydrogen absorption, a model suggested by Appenzeller and Hiltner (1967) for $\beta$ Lyr. A more detailed and quantitative model in which free-free emission also is significant has been given by Capps et al. (1973). These will be discussed in more detail in Section 5.

Reviews of polarization in circumstellar envelopes have been published by Zellner and Serkowski (1972) and by Kruszewski (1974). Both of these covered a wide variety of stars. While the same physical processes may explain the polarization observed in a wide variety of objects, there are specific characteristics of the polarization found in early-type emission-line stars. The aim of this review is to emphasize both those peculiar characteristics and the more recent developments, specifically the polarization changes across emission lines.

In separate sections we shall treat of the wavelength dependence of the continuum polarization (Section 2), the variability of the polarization (Section 3), the polarization changes across emission lines (Section 4) and models (Section 5). A summary of all available data on the variability of polarization in Be stars is given in Table I of Section 3, and in Section 4, Table II summarizes available information on all stars for which polarimetry in the $\mathrm{H} \alpha$ and $\mathrm{H} \beta$ emission lines has been measured.

\section{Wavelength Dependence of the Polarization in the Continuum Radiation of Be Stars}

The peculiar characteristics of the wide- and intermediate-band polarimetry in the visual regions of the spectrum of $\mathrm{Be}$ stars are: (1) a more rapid decrease in the 
POLARIZATION IN Be STARS

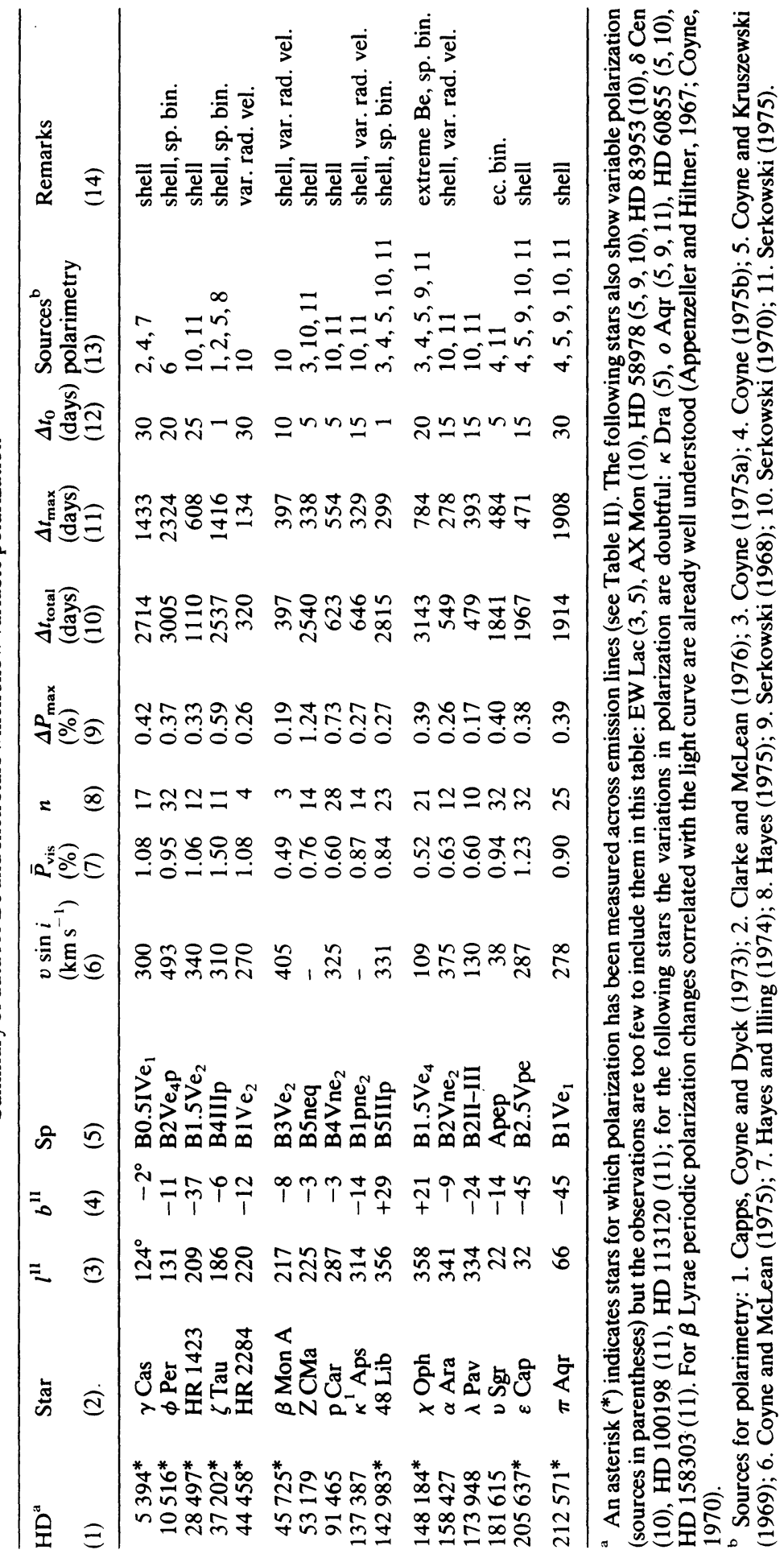


G. V. COYNE, S.J.

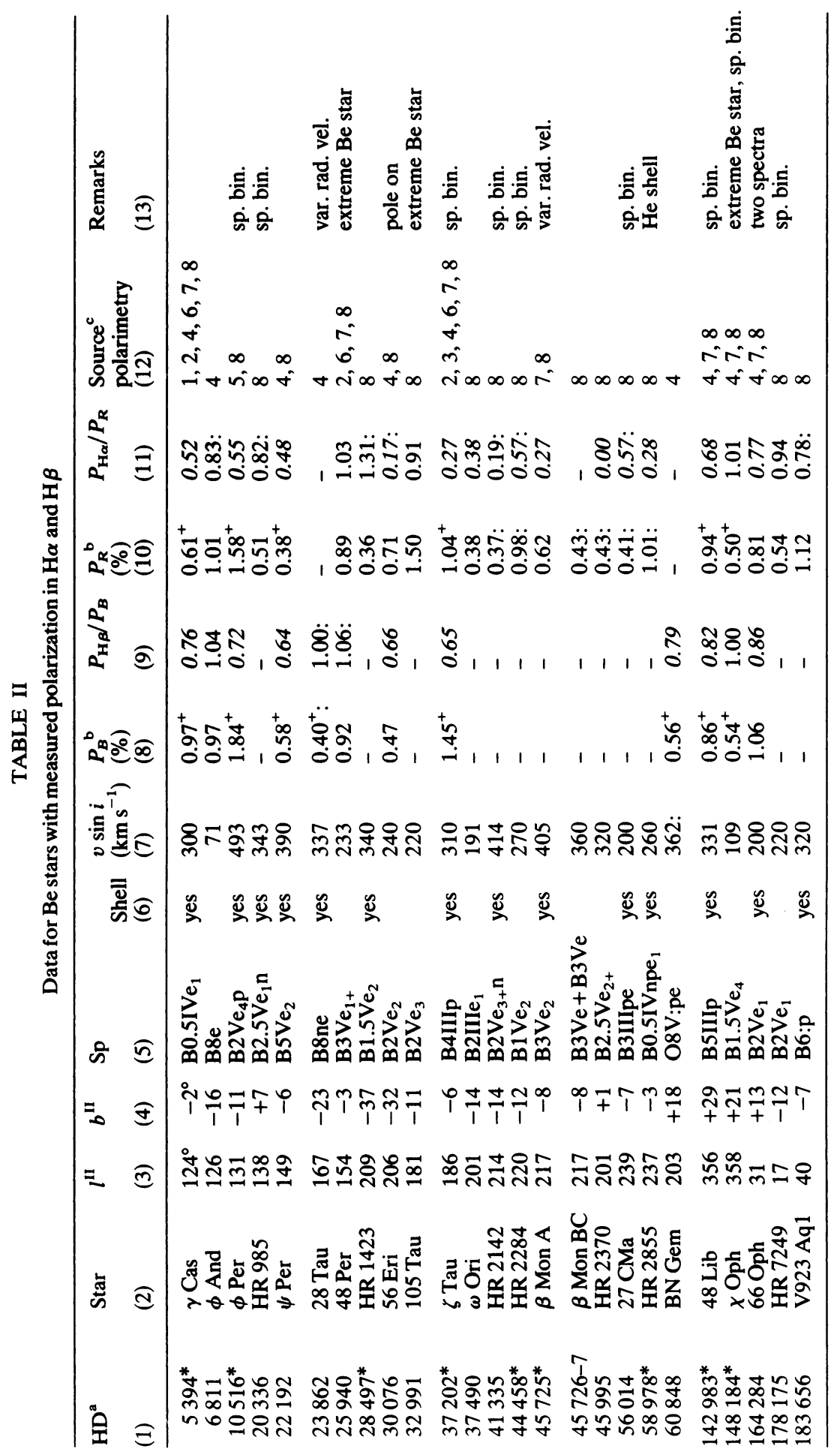




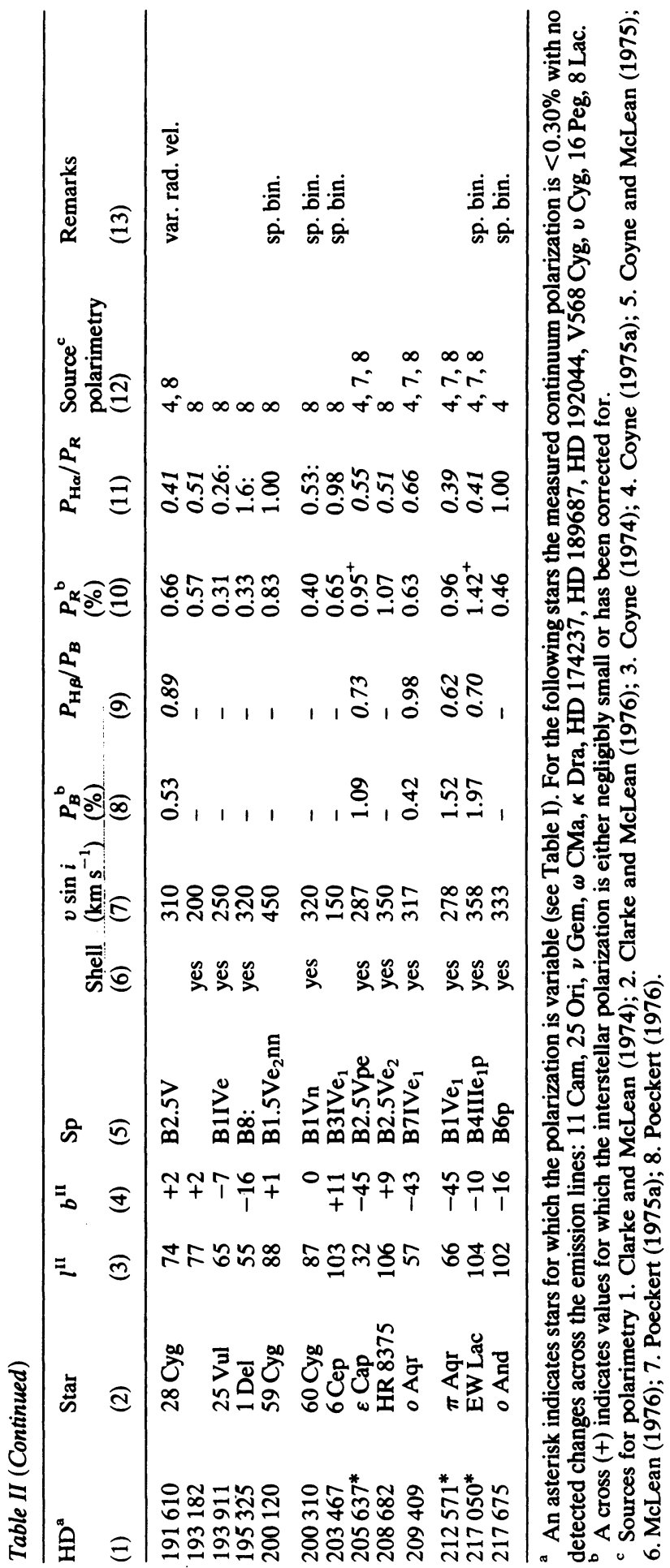


polarization towards the ultraviolet region of the spectrum; (2) a minimum polarization just to the blue side of the Paschen limit and an increase in the polarization to the red side of the Paschen limit. A few observations have been made at near infrared wavelengths (between 1 and $2.2 \mu \mathrm{m}$ ) and these indicate another decrease in the polarization after a maximum is reached in the $\lambda=1 \mu \mathrm{m}$ region.

The multicolor polarimetry done by Serkowski at the Mount Stromlo and Siding Spring Observatories of the Australian National University (e.g. Serkowski, 1968, 1970) and by a group at the Lunar and Planetary Laboratory of the University of Arizona (e.g. Coyne and Gehrels, 1967; Coyne and Kruszewski, 1969; Coyne et al., 1974) have provided the bulk of data on $p(\lambda)$ for the Be stars. Serkowski (1968) first noted the decrease in the ultraviolet polarization and remarked on the similarity to the polarization observed in several supergiant stars, in $\beta$ Lyrae, and in the recurrent nova T Pyxidis. The discovery by Serkowski and the prediction by Harrington and Collins (1968) of considerable polarization produced in the photospheres of rapidly rotating early-type stars led Coyne and Kruszewski (1969) to an analysis of multicolor polarimetry accumulated over a period of several years on 19 early-type stars with extended atmospheres. While considerable differences were seen from star to star (see Figure 1 of Coyne and Kruszewski 1969) eight of the stars observed showed the typical $p(\lambda)$ curve illustrated at the bottom of Figure 3 . Here the mean

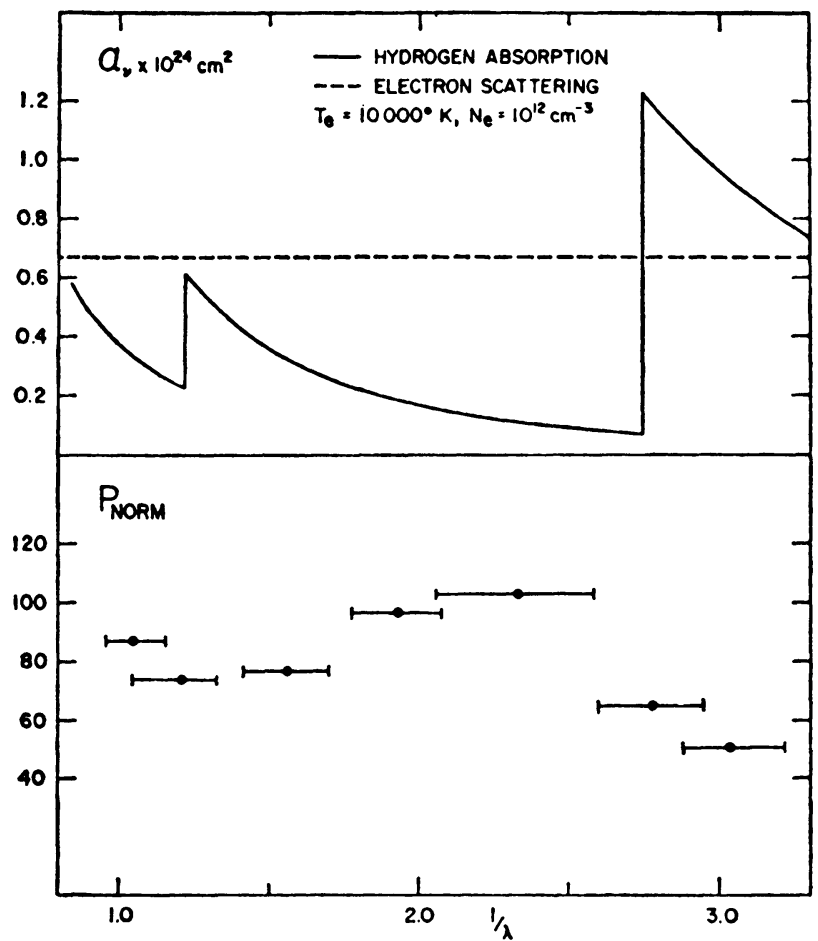

Fig. 3. At the bottom the mean normalized polarization for eight Be stars is plotted (the horizontal lines indicate the passbands); at the top the opacities for hydrogen absorption and electron scattering are compared. The polarization and hydrogen absorption are inversely related. 
polarization over the eight stars is given (after normalizing by equating to $100 \%$ the average polarization at the two intermediate filters). The horizontal bars indicate the passband. This curve indicated that hydrogen absorption played a significant role in the observed $p(\lambda)$. The hydrogen absorption coefficient is plotted as the solid line in the upper part of Figure 3 and the anti-correlation with the $p(\lambda)$ curve at the bottom is obvious. This suggested that polarization produced by electron scattering (independent, therefore, of wavelength) but modified by absorption in a hydrogen plasma both before and after scattering was responsible for the observed wavelength dependence. To produce such polarization it was necessary to have a hydrogen plasma characterized by such parameters as to make the electron-scattering opacity dominant at some wavelengths and the hydrogen absorption opacity dominant at others. At the top of Figure 3 a comparison of the two sources of opacity is given and we see that the condition is fulfilled for an electron temperature $T_{e}=10000 \mathrm{~K}$ and an electron density $N_{e}=10^{12} \mathrm{~cm}^{-3}$. It is also necessary, of course, that the scattering optical depth be sufficient to give the observed amount of polarization. We shall see in Section 5 that the path length required in the flattened hydrogen-plasma disk is about 3 to 10 stellar radii, consistent with what is otherwise known about these disks.

The rise of the polarization to the red side of the Paschen limit makes one curious about the polarization in the infrared region of the spectrum. Although such observations are difficult, since Be stars are relatively faint in the infrared, polarimetric observations of Be stars have been extended to $2.2 \mu \mathrm{m}$ by Dyck (Capps et al., 1973) for $\zeta$ Tau and by Vrba (Coyne and McLean, 1975) for $\phi$ Per. In both cases the polarization decreases in the infrared to a value at $2.2 \mu \mathrm{m}$ which is about one-half of that at $1 \mu \mathrm{m}$ where a maximum occurs just longwards of the Paschen limit. In the more quantitative treatment of the electron-scattering disk model (Capps et al., 1973) it is actually free-free emission which mainly governs the wavelength dependence of the polarization in the infrared. We shall discuss this in more detail in Section 5.

In the discussion above we have emphasized the characteristics of the $p(\lambda)$ curve which are common to all the intrinsically polarized Be stars which we have observed. It is necessary to emphasize that there are significant differences from star to star. These differences can be due to the fact that different stars are affected by differing amounts of interstellar polarization and/or to the fact that the intrinsic polarization differs from star to star. The intrinsic differences among various $\mathrm{Be}$ stars are illustrated by Figure 4 where the intrinsic polarizations for four Be stars are plotted along with a model calculation (solid curve) to be discussed in Section 5. For two of the stars, $\gamma$ Cas and $\zeta$ Tau, there is none or very little interstellar polarization (see Capps et al., 1973; Clarke and McLean, 1974); for $48 \mathrm{Lib}$ and $\psi$ Per the interstellar polarization has been removed (Coyne, 1975a). The differences among the stars are undoubtedly due to different electron temperatures and densities in the extended atmospheres of these stars.

It is obviously of critical importance, whenever possible, to recover the intrinsic polarization from the observed polarization by removing the interstellar effects. We should, therefore, like to consider the ways in which this is done.

The interstellar polarization for these objects is not directly known. A clue to its presence is the rotation of the plane of the observed polarization with wavelength. 


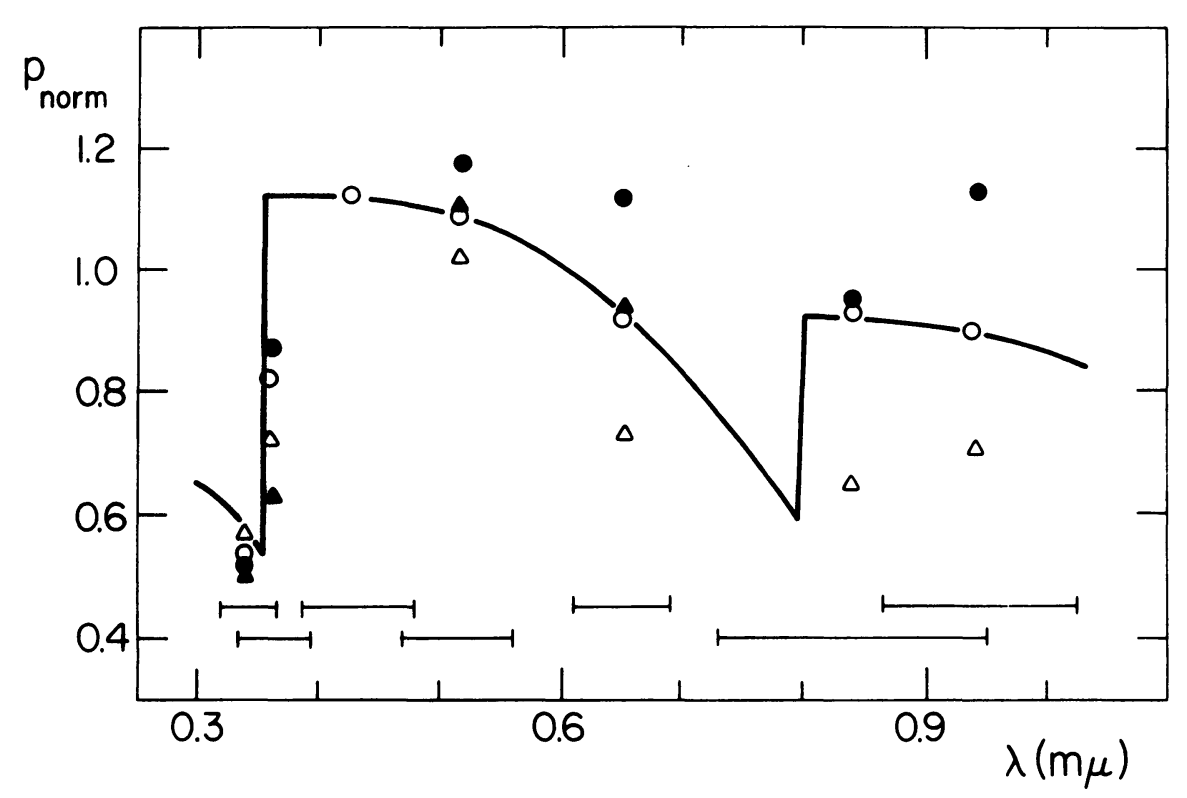

Fig. 4. The normalized polarization for four Be stars (solid circle: $48 \mathrm{Lib}$, solid triangle: $\psi$ Per, open circle: $\zeta$ Tau, open triangle: $\gamma$ Cas) are compared to a model calculation (solid line, see Section 5).

One criterion, therefore, for indirectly determining the interstellar polarization is that, when the interstellar effects are removed from the observations, a mean plane of polarization independent of wavelength is found. The amount of interstellar polarization and its orientation may be inferred from observation of stars in the surrounding region. One is, of course, then assuming a certain homogeneity of the interstellar effects over this region, an assumption which is not always verified. The following sources are useful for determining the degree of homogeneity of interstellar polarization effects: Hall (1958); Hiltner (1956); Serkowski et al. (1975); Coyne et al. (1974). In the latter two sources the wavelength dependence of the polarization characteristic for different regions of the Galaxy is given in terms of $P_{\max }$ and $\lambda_{\max }$, the maximum polarization and the wavelength at which it occurs. The interstellar polarization at various wavelengths is then given by the formula

$$
p(\lambda)=p_{\max } \exp \left(-1.15 \ln ^{2} \lambda_{\max } / \lambda\right) .
$$

Serkowski (1970) first showed that the interstellar polarization could be determined graphically if one assumed a certain wavelength dependence for the intrinsic and for the interstellar polarization and also assumed that at any given time the position angle of the interstellar component and the intrinsic component were independent of wavelength. Since we now know that even among the Be stars the intrinsic polarization varies somewhat from star to star, it would be preferable not to assume the $p(\lambda)$ of the intrinsic polarization. For $\phi$ Per Coyne and McLean (1975) successfully removed the interstellar effects without making any assumptions about the intrinsic polarization except that the plane of the intrinsic polarization be independent of wavelength. The results are shown graphically in Figure 5 where for 


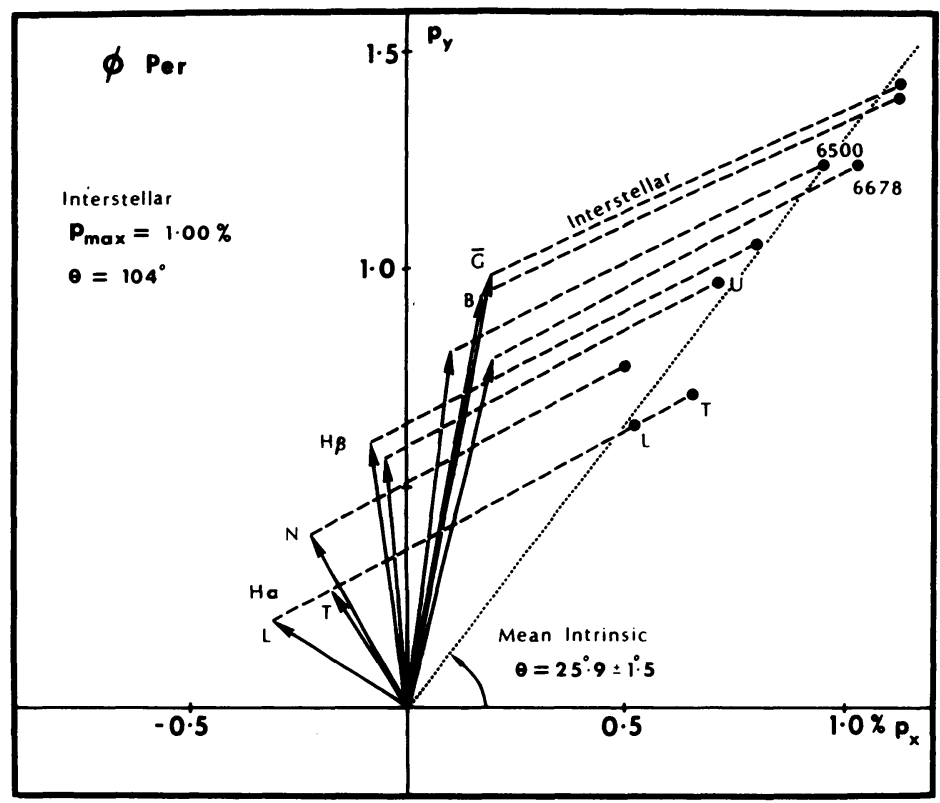

Fig. 5. The observed (arrows), interstellar (dashed line) and intrinsic (solid circles) polarization are plotted in polar co-ordinates for $\phi$ Per. A well defined direction for the intrinsic polarization is obtained.

$\phi$ Per the observed, interstellar and resultant intrinsic polarizations at various wavelengths are plotted in polar coordinates. One sees that, except for observational scatter, the resultant plane of the intrinsic polarization is independent of wavelength. The vectors marked $H \alpha$ and $H \beta$ represent polarizations measured with narrowband interference filters centered on the emission lines. We shall discuss these in Section 4 , but wish now to note the following. If the plane of the intrinsic polarization is the same for the emission line as for the continuum, then independent of any assumptions concerning the interstellar polarization the following must be true. The line which joins the line center polarization in $\mathrm{H} \alpha$ to that for the nearby continuum ( $\lambda 6500 \AA$ in Figure 5) must be parallel to the corresponding line for $\mathrm{H} \beta$ (continuum measurement is $B$ in Figure 5) and this line defines the direction of the intrinsic polarization. In Figure 5 it is clear that the two lines are indeed parallel and their position angles ( $26^{\circ}: 0$ and $26^{\circ} .3$ respectively for $\mathrm{H} \alpha$ and $\mathrm{H} \beta$ ) are exceedingly close to the mean value of $25^{\circ} 9 \pm 1.5$ derived by removing the interstellar polarization component. This strengthens the conviction that the interstellar component has been correctly estimated. In the same way Coyne (1975a) has been able to extract the intrinsic polarization for several other Be stars plotted in Figure 4. Furthermore, Poeckert $(1975 \mathrm{a}, \mathrm{b})$ has shown that if one can assume a certain relationship governing the change in the polarization across the emission line (e.g., that the change is due to unpolarized emission flux) then one can determine the magnitude and direction of the interstellar component from the line and continuum polarization alone. McLean (1976) has shown that both the interstellar and intrinsic components of the polarization can be determined from measurements at two unpolarized 
emission lines. He also discusses how the intrinsic polarization can be determined if the polarization is variable. It is fortunate that in many cases of Be stars we can successfully remove the interstellar effects or at least make a fair estimate of them. Otherwise an analysis of the observations would be difficult. The observed polarization in Be stars is not greater than $2 \%$ and a significant fraction of that may be due to interstellar polarization, since the Be stars are concentrated to the galactic plane.

\section{Variability of the Polarization}

About $40 \%$ of all Be stars which have been observed polarimetrically more than one time with mean errors less than $\pm 0.05 \%$ show changes of polarization with time. It is likely that many more Be stars would show variable polarization if the polarimetric observations were made when spectral changes were known to be occurring. No such systematic study has yet been undertaken. Feinstein $(1968,1976)$ reports small amplitude photometric changes (generally less than $0^{m} 15$ ) in Be stars but no general correlation of brightness and polarimetric changes in the Be stars has been established. Several of the most frequently observed Be stars are members of binary systems. For instance, $\zeta$ Tau, $\phi$ Per and $48 \mathrm{Lib}$ are spectroscopic binaries, $v$ Sgr is an eclipsing binary. All show polarimetric changes with time but there is no correlation with either the radial velocity or light curves, as the case may be, despite the fact that in the case of $\zeta$ Tau, $\phi$ Per and $v$ Sgr observations were made specifically to detect such correlations (Coyne, 1975b). Although in general no systematic study has been made of wide-band polarimetric changes in Be stars in such wise that correlations with other observational parameters, and especially spectral changes, could be sought, it would be useful from existing data to summarize what we do know of the polarimetric changes in these stars. Table $I$ is a summary of polarimetric changes in Be and shell stars based on observations made over the past decade mainly by the Australian and Arizona groups mentioned in the Introduction. There appear to be no serious systematic effects between these two groups of observations (see Coyne et al., 1974; Serkowski et al., 1975). In order to avoid inhomogeneities and because, for our purposes, it was not necessary to have a longer baseline in time we have not included here the polarimetric observations antecedent to the past decade by Behr (1959), Hall (1958), and Hiltner (1956). The sources used are given in Table I. Columns (1) to (6) give the star identification, galactic coordinates, spectral type and projected rotational velocity. The remaining columns summarize the polarimetric data. The spectral types are for the most part from Lesh (1968). The values of $v \sin i$ are from Bernacca and Perinotto (1971) except for a few values from Boyarchuk and Kopylov (1964) or Uesugi and Fukuda (1970). The average polarization in the visual spectral region, $\bar{P}_{\text {vis }}$, the total number of observations in the visual spectral region, $n$, the maximum observed change in the visual polarization, $\Delta P_{\max }$, are given in columns (7) to (9). The visual polarizations are the ' $V$ ' observations $\left(\lambda_{0} \cong 0.57 \mu \mathrm{m}\right)$ of Serkowski (Sources No. 9, 10 and 11 in Table I), the ' $G$ ' observations $\left(\lambda_{0} \cong\right.$ $0.53 \mu \mathrm{m}$ ) of Coyne (Sources No. 1, 3, 4, 5, 6 in Table I) and for the few observations from other observers in Table $I$ those observations which are closest in effective wavelength to these. For our purposes the difference in the effective wavelengths of the ' $V$ ' and ' $G$ ' filters is insignificant. The values of $\Delta t$ in columns (10) to (12) are 
intervals of time over which respectively the total number of observations were made $\left(\Delta t_{\text {total }}\right)$, over which the maximum change in polarization occurred $\left(\Delta t_{\text {max }}\right)$, and over which the polarization changed by more than $0.15 \%\left(\Delta t_{0}\right)$, a value greater in general than three times the standard deviation of a single observation. The values of $\Delta t_{0}$ are rounded off to the nearest five days except for cases where there was clearly a change over a period of one day or less. Because the observations are so non-uniformly scattered in time, no periodicity studies are intended by these intervals of time. They give, however, an idea of the time scale over which significant polarization changes take place.

With the exception of $\mathrm{Z} \mathrm{CMa}$ and $\mathrm{p}$ Car the amplitude of the polarization change, $\Delta P_{\max }$, is on the average $40 \%$ of the mean value of the polarization. For $\mathrm{Z} \mathrm{CMa}$ and p Car the amplitude of the polarization change is larger than the mean polarization itself. For a given star the observations have extended over a time ranging from one to eight years during which in general a polarization maximum and minimum have been observed several times. The maximum observed change in polarization has been observed over a time interval significantly shorter in general than the total observing time. Merrill (1956) has shown that the shell typically appears and disappears with a period of about 8 to 10 years. The maximum changes in polarization apparently occur in a much shorter interval of time.

The typical polarization changes for the Be stars can be described as follows. There are apparently longer time scale changes over intervals of some hundreds of days upon which there are superimposed changes on a time scale of days. An example of this is Figure 6 (from Zellner and Serkowski, 1972) where the polarimetric observations in two spectral regions for $\mathrm{p}$ Car are plotted. This star is little, if at all, affected by interstellar polarization (Serkowski, 1970).

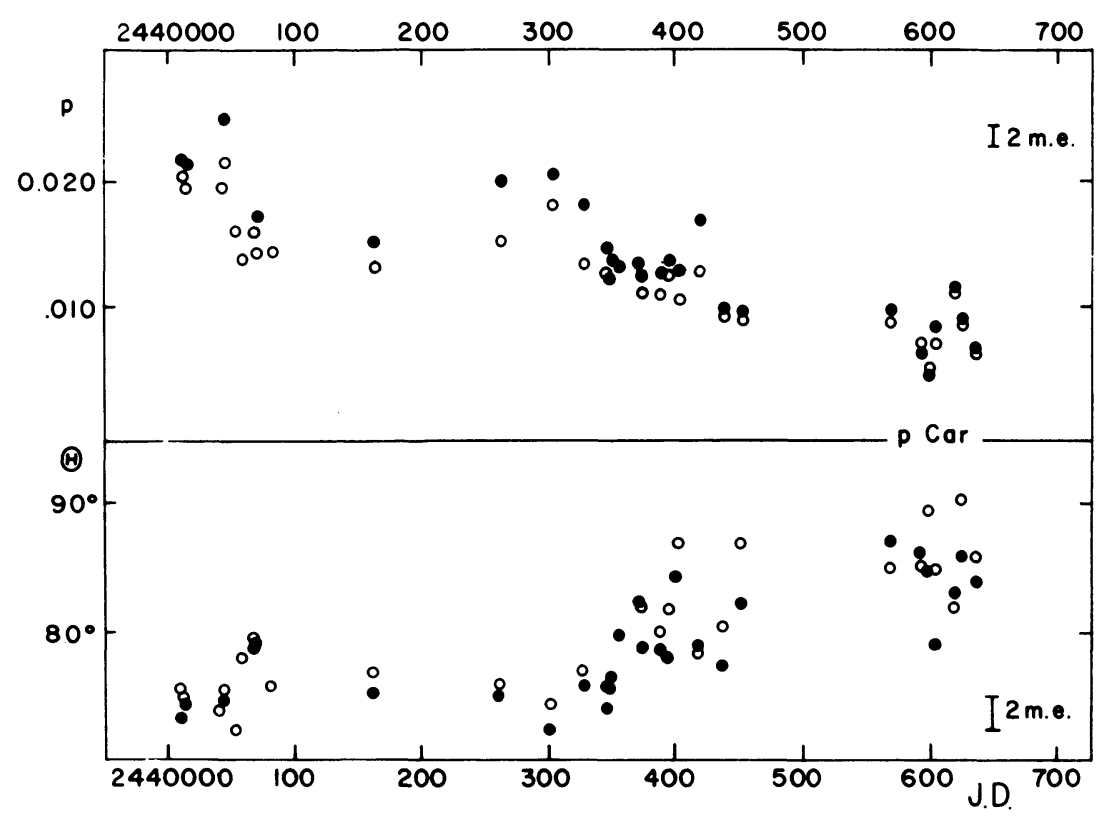

Fig. 6. Polarization (in magnitudes) for $\mathrm{p}$ Car in two spectral regions is plotted vs time. 
We see from column (12) of Table I that for all of these stars significant changes in the polarization take place over an interval of days. The value of $\Delta t_{0}$ would undoubtedly be shortened for many of these stars if more frequent and systematic observations were made. The change over a one day interval for $48 \mathrm{Lib}$ is obtained from observations by Serkowski (1970) during the days JD 2440043-45. A change of $\Delta P=0.3 \%$ over an interval of 24 hours has been measured for $\zeta$ Tau by Poeckert (1975a). Clarke and McLean (1976) report a change of $\sim 0.2 \%$ over an interval of 40 minutes for the polarization in the continuum at $\lambda 4837$ in $\zeta$ Tau.

For 10 of the stars in Table I (marked with an asterisk in column 1), narrow-band measures of the polarization across the $\mathrm{H} \alpha$ and $\mathrm{H} \beta$ emission lines have been made (see Table II, Section 4). All except $\chi$ Oph and HD 28497 also show changes in polarization across the emission lines. $\chi$ Oph is an extreme Be star (Schild, 1966, 1973). It is a noteworthy case and we shall return to a discussion of it in Section 4. It is difficult to see how these changes in polarization, especially the short interval ones, can be attributed to processes occurring in the stellar photosphere. They are an independent indication that the polarization must be due to processes in an extended circumstellar disk.

\section{Polarization Measurements in the Hydrogen Emission Lines}

Since the continuum and the emission-line flux originate at different levels in the atmosphere and extended flattened disk about Be stars, one would predict that the polarization produced in the circumstellar regions would vary across the emission lines. In particular, the continuum flux will presumably be scattered through the total path length of the extended atmosphere; the emission flux, originating in the extended atmosphere itself, will be scattered through only a part of that path length. The polarization should, therefore, decrease in the emission lines depending on the relative strength and polarization of the emission to continuum flux. Such changes in polarization across lines are clear proof that at least part of the observed polarization arises in the circumstellar region and an analysis of the changes would assist in determining the physical parameters of the circumstellar region, viz. the electron temperature and density, scattering optical depth and thus the linear dimensions of the circumstellar shell, mean depth at which the emission flux originates, etc.

In recent years there have been a number of studies dedicated to polarimetry in the emission lines of $\mathrm{Be}$ and shell stars. In the Introduction, we have reviewed the history of the beginnings of these studies. Most of the observations have been done on the brightest stars at the $\mathrm{H} \alpha$ and $\mathrm{H} \beta$ lines. Some measurements have been made at the $\mathrm{H} \boldsymbol{\gamma}$ line (Hayes and Illing, 1974; Hayes, 1975). Current instrumentation is flux limited and it has not been possible to observe fainter stars or the higher lines in the Balmer series. The general technique is to scan across the emission lines by tilting a narrow-band interference filter to various discrete positions and the spectral resolutions obtained vary between 2 and $12 \AA$. Since the equivalent width in many Be stars is less than $10 \AA$, the lines are instrumentally broadened by the wider of these filters. Descriptions of the various polarimeters used are given in the literature (Angel and Landstreet, 1970a, b; Illing, 1973; Clarke and McLean, 1975; Clarke et al., 1975; 
Poeckert, 1975a). Coyne (Coyne, 1975a, b; Coyne and McLean, 1975) used wider interference filters (passbands of 8 and $26 \AA$ respectively at $\mathrm{H} \beta$ and $\mathrm{H} \alpha$ ) and did not tilt them. All investigators simultaneously or quasi-simultaneously measure the continuum at appropriate wavelengths near the emission lines.

The analysis of the polarization measured in the emission line and in the continuum is done in the following way. If both the continuum flux and the emission flux are polarized in the same plane or orthogonal to one another, the following general relation can be written:

$$
p_{L}=\frac{p_{A}+p_{E} X}{1+X}
$$

where $p_{L}$ is the observed polarization in the line, $p_{A}$ the polarization at the same wavelength in the underlying absorption, $p_{E}$ the polarization of the emission flux and $X$ the intensity ratio of the emission flux to that in the underlying absorption. In practice $p_{A}$ is taken as $p_{C}$, the polarization in the adjoining continuum. If the underlying absorption is small with respect to the emission flux then $X$ may be taken as the ratio of the emission flux to the continuum flux. It is customary to take the continuum flux as unity. Then $1+X=I$, the total intensity of the emission line. With these assumptions, the simplest case is when the emission flux is unpolarized, $p_{E}=0$ and $p_{L}=p_{C} / I$. If, in other words, the observed $p(\lambda)$ across the emission line varies inversely as $I(\lambda)$ the emission flux is unpolarized. Any deviation from this dependence will indicate either that $p_{E} \neq 0$ or that Equation (1) is not applicable.

In general the observed values of $p_{L}$ and $p_{A}$ or $p_{C}$ include interstellar polarization effects. Poeckert (1976) has assumed $p_{L}=p_{C} / I$ in order to determine the interstellar polarization. Elsewhere in this Symposium McLean (1976) discusses ways in which the observations of polarization in emission lines together with Equation (1) can be used to separate out the interstellar and intrinsic effects and I shall not discuss this more here. McLean (1976) also shows how $p_{E}$ may be determined from polarimetric and photometric measurements at two different wavelengths within an emission line.

Examples of the results obtained are shown in Figures 7 and 8 taken respectively from Coyne (1975a) and Poeckert (1975a). In Figure 7 is shown for six stars the variation of $p$ and $\theta$ with wavelength. The measurements at $\mathrm{H} \alpha$ and $\mathrm{H} \beta$ were made with filters having passbands of 26 and $8 \AA$ respectively. The wide and intermediateband filters used at the other wavelengths are described by Coyne (1975a). o Sco is not a Be star but is a standard highly polarized star and it shows the normal interstellar polarization curve. All other stars show a decrease in the polarization at $\mathrm{H} \alpha$ and $\mathrm{H} \beta$. For $48 \mathrm{Lib}$ and $\psi$ Per there is a dependence of $\theta$ on wavelength. Coyne (1975a) has shown that this is due to a combination of the intrinsic and interstellar polarization and has used the effect to remove the interstellar polarization. In Figure 8 we have high resolution $(\sim 2 \AA)$ polarimetric and line profile measurements across the $\mathrm{H} \alpha$ line of $\gamma$ Cas. Compare this to similar measurements by McLean (1976, page 263 , Figure 1d). It is clear from both sets of measurements that the polarization in the blue wing of the $\mathrm{H} \alpha$ line is less than obtainable from the simple addition of unpolarized emission flux. Some other mechanism must be causing a decrease in the polarization here. Other stars observed at high resolution also show anomalous polarimetric changes across the emission line, i.e. changes which cannot be explained 


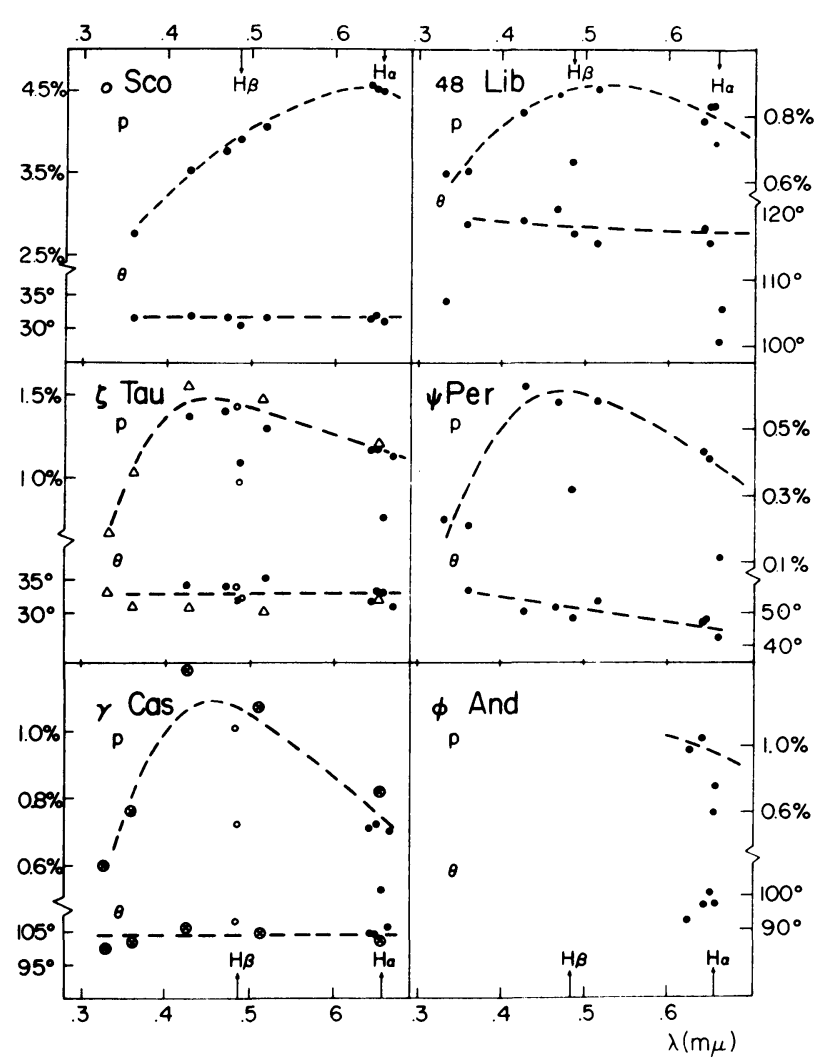

Fig. 7. A decrease in polarization across the emission lines $\mathrm{H} \alpha$ and $\mathrm{H} \beta$ are shown for five Be stars. $o$ Sco is a typical interstellar polarization star.

by the simple addition of unpolarized emission flux. We shall discuss the more important cases shortly but first we wish to summarize the information available for all Be stars for which polarization has been measured across the $\mathrm{H} \alpha$ and/or $\mathrm{H} \beta$ emission lines.

Table II presents the essential data obtained from narrow-band polarimetry of Be and shell stars. Columns (1) to (7) give the same fundamental data as in Table I. In column (6) we have attempted to identify all of these stars which have ever shown shell characteristics. Since shell characteristics are transient phenomena and an exhaustive literature search was not possible, this column may be incomplete. The sources for the spectral types and $v \sin i$ are the same as described for Table $\mathrm{I}$. The remaining columns summarize the polarimetric results. Columns $(8)$ and (10) list the mean polarization values for the continuum in the Blue region of the spectrum near $\mathrm{H} \beta$ and the Red region of the spectrum near $\mathrm{H} \alpha$ respectively. The exact wavelength at which the continuum polarization is sampled differs from author to author but has no effect on these results since $p(\lambda)$ for the continuum near the $\mathrm{H} \alpha$ and $\mathrm{H} \beta$ lines does not vary rapidly with $\lambda$. The ratios of the polarization in the emission line to that of the continuum are given in columns (9) and (11). The sources of the polarimetry are 


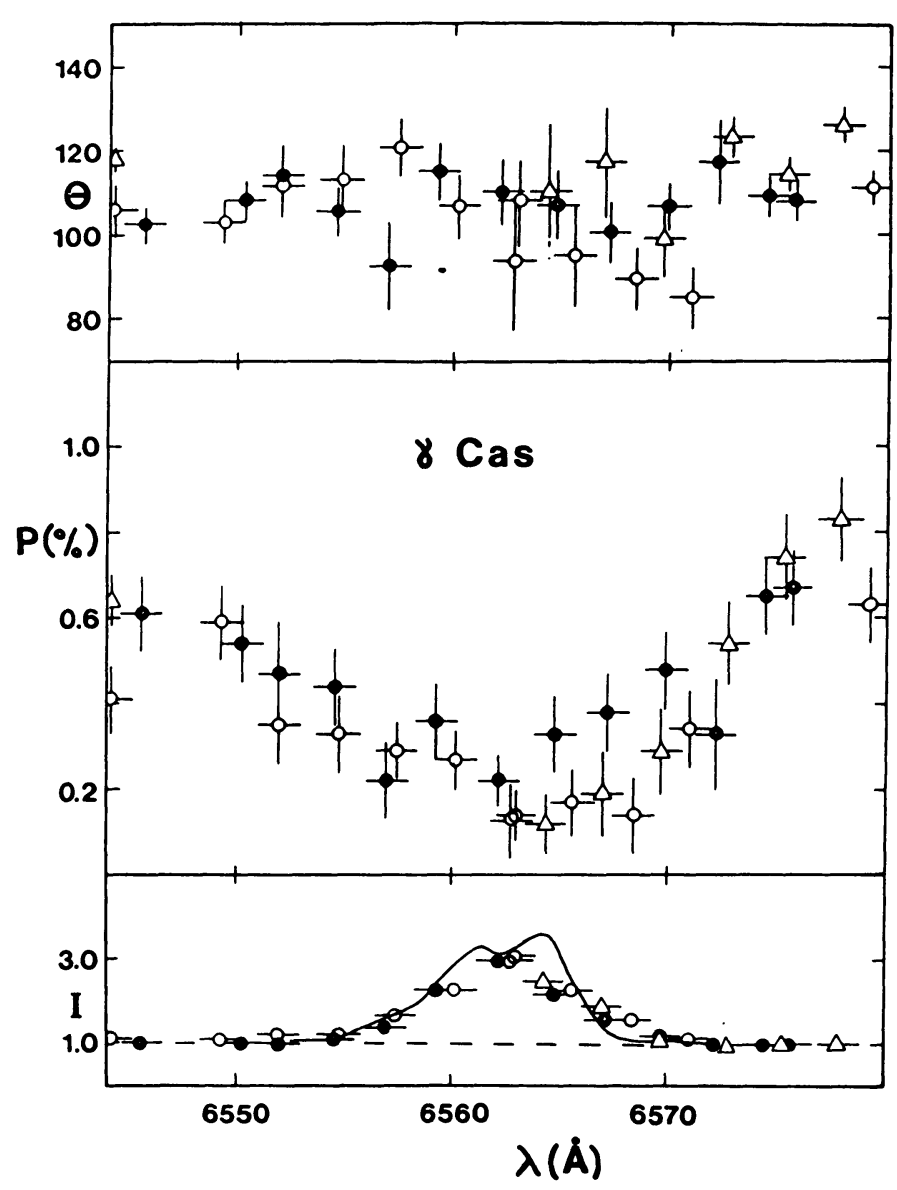

Fig. 8. The direction and percentage of polarization and the intensity profile across $\mathrm{H} \alpha$ are shown for $\gamma$ Cas.

listed in column (12). We have omitted cases where the measured continuum polarization was less than $0.3 \%$ or where the observational errors were too large to determine the polarization ratios. The average error for a polarization measurement is $\pm 0.05 \%$. Colons in columns (8) and (10) indicate errors greater than $\pm 0.1 \%$.

Of the 39 stars investigated 21 show significant changes [the polarization ratios are in italics in columns (9) and (11)] in polarization across $\mathrm{H} \alpha$ and /or $\mathrm{H} \beta$. These have a mean value of $v \sin i$ of $303 \mathrm{~km} \mathrm{~s}^{-1}$. For seven stars which do not show changes in polarization across the emission lines the mean value of $v \sin i$ is $203 \mathrm{~km} \mathrm{~s}^{-1}$. The star 59 Cyg is an anomaly with $v \sin i=450 \mathrm{~km} \mathrm{~s}^{-1}$ and no polarization changes across the emission line. It is omitted from the means mentioned above. With the possible exception of 1 Del and $o$ And all stars which are identified as shell stars and which are measured with sufficient accuracy show polarization changes across the emission lines. There are five stars which show polarization changes across the emission lines but are not listed as shell stars (56 Eri, $\omega$ Ori, HD 45995, BN Gem and 28 Cyg). 
None of the three extreme Be stars (see Remarks column (13) of Table II) of Schild (1966, 1973) shows polarization effects across the emission lines. Three other extreme Be stars with continuum polarization less than $0.3 \%$ (not included, therefore, in Table II) showed no decrease of polarization in the emission lines. It appears, therefore, from the limited sample of stars observed to date that shell characteristics are a sufficient but not a necessary condition for the existence of decreased polarization across the emission lines and that extreme Be stars show no such effects.

For a number of stars, the decrease in polarization across the emission lines can be explained simply by the addition of unpolarized emission flux to the continuum polarization. According to Equation (1) this means that, after the removal, if necessary, of interstellar polarization effects, the polarization varies as $1 / I$.

Figures 9 and 10, from Poeckert (1975a), give examples of cases where for $\zeta$ Tau and $48 \mathrm{Lib}$ respectively the polarization at the center of the $\mathrm{H} \alpha$ emission line varies as $1 / I$. In the case of $\zeta$ Tau, which has little or no interstellar polarization, although the level of the continuum polarization varies (at times over a period of one day or less), the dependence on $I$ remains the same. In other words the emission flux is, apparently, always unpolarized. The case with $48 \mathrm{Lib}$ is different, since there is significant interstellar polarization for this star. Poeckert (1975a) assumes that the

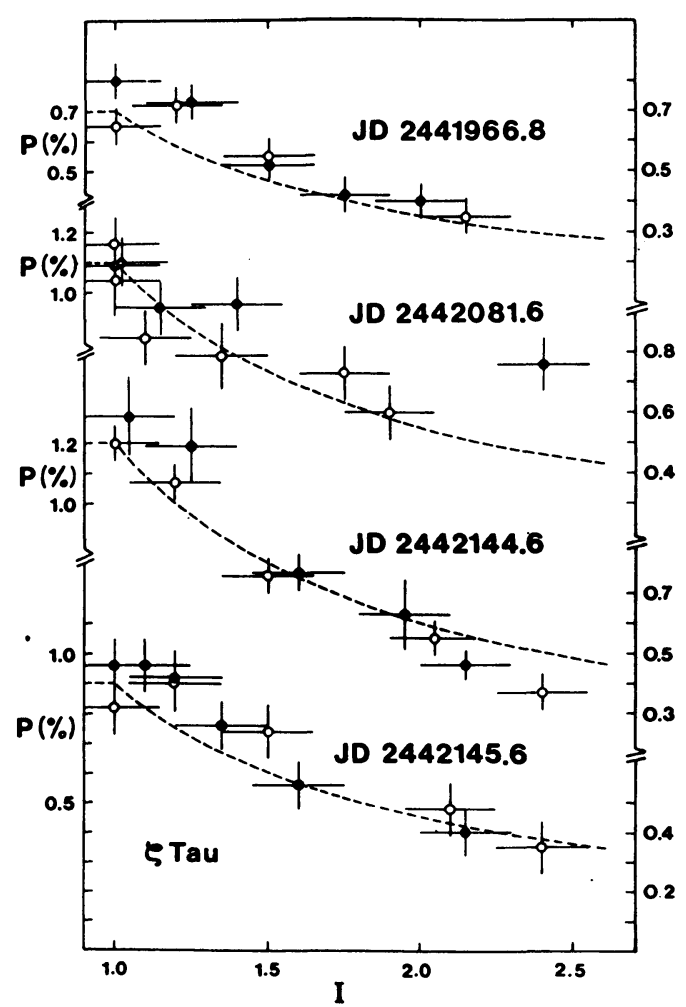

Fig. 9. The variation of polarization as a function of the intensity across the H $\alpha$ emission line of $\zeta$ Tau. 


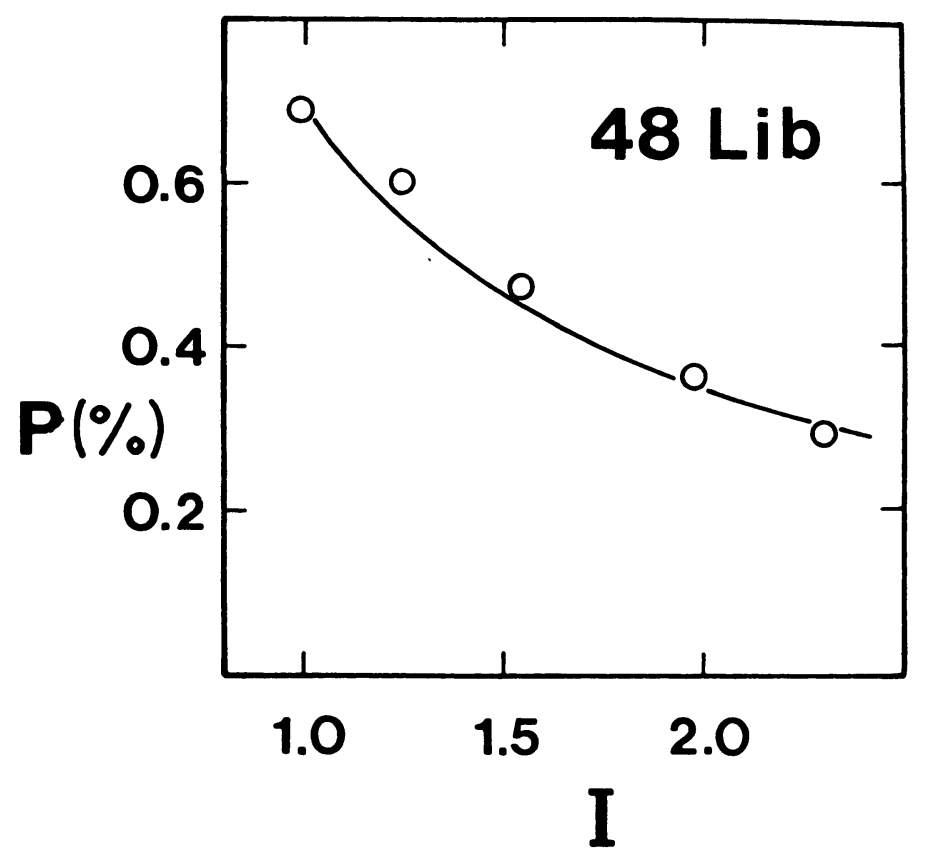

Fig. 10. The variation of polarization as a function of the intensity across the $\mathrm{H} \alpha$ emission line of $48 \mathrm{Lib}$. The interstellar polarization has been removed.

continuum polarization in $48 \mathrm{Lib}$ will vary as $1 / I$ and uses this relationship to remove the interstellar components. Coyne (1975a) has shown that the interstellar polarization derived by Poeckert is essentially correct and results in a well-defined direction for the intrinsic polarization. Thus in Figure 10 the dependence of the polarization on $1 / I$ represents the intrinsic polarization in $48 \mathrm{Lib}$ and shows that the emission flux is essentially unpolarized. Other stars for which the interstellar polarization is either very small or has been corrected for in the manner of $48 \mathrm{Lib}$ are indicated by a cross $(+)$ in column (8) and/or (10) in Table II.

However, the addition of unpolarized emission flux does not appear to be adequate explanation for all stars. For some stars there appears to be a residual polarization after account is taken of the unpolarized emission flux. For instance, for $\phi$ Per it appears that the emission flux, at least in $\mathrm{H} \alpha$, may be polarized about $0.5 \%$ (Coyne and McLean, 1975; McLean, 1976). If the emission flux is polarized as it is in $\phi$ Per it probably occurs by scattering if there is sufficient scattering optical depth. For $\phi$ Per these conditions are probably fulfilled. It is likely as a matter of fact that we are viewing the disk edge on; $v \sin i$ for $\phi P e r$ is $493 \mathrm{~km} \mathrm{~s}^{-1}$. Coyne and McLean (1975) derive a scattering optical depth of $\tau=0.04$ corresponding to a path length of one-half a stellar radius in their simplified model of $\phi$ Per.

One would like to know from the body of data we now have (see Tables I and II) whether the values found for the polarization in the emission lines of Be stars can be typically explained by the simple model of the addition of unpolarized emission flux or whether other mechanisms must be considered such as suggested for $\phi$ Per. 
Unfortunately simultaneous or quasi-simultaneous photometry and polarimetry are not available for all of the stars of Table II. Furthermore a correct estimate of $I$ from the line profile is difficult. McLean (1976) makes the interesting suggestion that additional shell absorption is responsible for an incorrect estimate of $I$ in the equation $p / p_{C}=1 / I$ in the sense that $I$ is really larger than measured, because the shell absorption, occurring in a layer further out in the extended atmosphere than the emission layer, affects only the intensity profile. In reality the failure to recognize both this shell absorption and the underlying atmospheric absorption may be responsible for an incorrect estimate of $I$. Nonetheless it appears that for some stars the emission flux is partially polarized. In the model to be proposed in Section 5 it is not unlikely that this is the case. All that is required is that the emission flux originate at a sufficiently large scattering optical depth in the extended atmosphere. The scattering optical depth for the continuum radiation would be, of course, considerably larger. Alternatively the polarization in the emission flux may be due to resonant scattering.

The differences between the amount of polarization in the emission flux for different stars are probably a function of aspect (when the observer lies in the orbital plane the optical depth is larger) but intrinsic differences between stars cannot be ruled out. Poeckert (1976) has attempted to determine the intrinsic polarization in Be stars by assuming that $p_{L}=p_{C} / I$ and then relating the intrinsic polarization to $v \sin i$. He finds only that for $v \sin i \leq 150 \mathrm{~km} \mathrm{~s}^{-1}$ the polarization is less than $0.2 \%$. For $v \sin i>150 \mathrm{~km} \mathrm{~s}^{-1}$ there is a large scatter in the polarization.

Essentially what we have spoken of so far is the central part of the polarimetric profile of the emission lines, i.e. the polarization corresponding to the center of the emission line. High resolution polarimetry across the whole line profile has revealed interesting results for several stars. In the blue wing of the $\mathrm{H} \alpha$ line in $\gamma$ Cas and extending into the continuum, the polarization is at times lower (McLean, 1976; Poeckert, 1975a) and at times higher (Poeckert, 1975b) than that expected from unpolarized emission flux. Three other stars, $\beta$ Mon A (Poeckert, 1975a), EW Lac (Poeckert, 1975b) and $\zeta$ Tau (McLean, 1976), have shown polarizations larger than the continuum polarization in the blue wing of the $\mathrm{H} \alpha$ line. McLean (1976) suggests that this increase in polarization in the line wings is due either to further electron scattering or to partially polarized flux from the underlying star itself. With respect to the first possibility others have suggested that the broad emission wings in Be stars are due to electron scattering; with respect to the second see the results from the model calculations for rigidly rotating flattened stars by Cassinelli and Haisch (1974) and Haisch and Cassinelli (1976).

In summary a comparison of polarimetric and photometric line profiles for Be stars suggests the following conclusions:

(1) All shell stars show a decrease in the polarization across emission lines.

(2) The emission flux is less polarized than the continuum flux, the ratio depends both on aspect angle and on particular characteristics of individual stars.

(3) Because of possible shell absorption superimposed on the emission and because of uncertainties about the underlying absorption it is difficult to determine the correct value of $I$, the total intensity at the emission line center normalized to the intensity of the underlying absorption. 
(4) The blue wing of the $\mathrm{H} \alpha$ emission line in some stars shows peculiar polarization effects.

There is one anomalous case. $\chi$ Oph is a 'pole-on' star (Slettebak and Howard, 1955) and an extreme Be star (Schild, 1973). The polarization is variable (see Table I) with a mean value in the visual of $0.52 \%$ and an amplitude of $0.39 \%$ and is, therefore, undoubtedly intrinsic. It shows, however, no polarization differences across the $\mathrm{H} \alpha$ and $\mathrm{H} \beta$ lines although the respective line intensities relative to the continuum are 18.5 and 4.28 respectively (Gray and Marlborough, 1974). Coyne (1975a) measured the continuum polarization to be $0.53 \% \pm 0.02$ with no measurable dependence on $\lambda$ between 0.36 and $0.66 \mu \mathrm{m}$. Thus if unpolarized emission flux were added to polarized continuum radiation then the measured polarization should be $0.03 \%$ and $0.12 \%$ at $\mathrm{H} \alpha$ and $\mathrm{H} \beta$ respectively. None of the other extreme Be stars or 'pole-on' stars observed (48 Per, $11 \mathrm{Cam}, 105 \mathrm{Tau}, \nu \mathrm{Gem}, \omega \mathrm{CMa}, v$ Cyg) show either variable polarization (only 48 Per has a large number of observations) or polarization effects across the emission lines. Besides $\chi$ Oph only two of these (48 Per and $105 \mathrm{Tau}$ ) are polarized more than $0.25 \%$. All other stars which show variable polarization and have been measured in the emission lines show polarization effects across these lines. The observations of $\chi$ Oph may be partially explained as follows. $\chi$ Oph is a conspicuous example of the stars identified by Schild (1973) as extreme Be stars. It has the strongest hydrogen emission lines among these stars and has shown little intensity variation in the emission lines (contrasted to the classical Be stars) over a period of 50 years. It has, compared to the classical Be stars, strong infrared excess $\left(E_{V-L}=0.52\right)$. It differs from other extreme Be stars, including all of those identified in Table II, and those enumerated above in the following two respects: (1) it has a lower $v \sin i$ than is to be expected from the relation of $v \sin i$ to the separation of emission line components for extreme Be stars (see Figure 3 of Schild, 1973); (2) whereas all extreme Be stars are optically thin in the hydrogen lines, $\chi$ Oph is optically thick (Burbidge and Burbidge, 1953).

We propose that $\chi$ Oph is an intermediate case. It is an extreme Be star but has the classical Be star characteristic of a somewhat flattened extended disk. As contrasted to the other extreme Be stars, $\chi$ Oph shows intrinsic polarization because it is optically thick. The polarization is neutral in the wide-band filters and shows no effect across emission lines because we are not viewing equator-on but at a sufficiently large angle of inclination $\left(i \cong 45^{\circ}\right.$ would remove the discrepancy in Figure 3 of Schild, 1973) that the path length for electron scattering for the emission and continuum flux through a flattened tilted disk are about the same but the path length is not large enough to introduce the processes responsible for the $p(\lambda)$ curve in shell stars (Coyne and Kruszewski, 1969; Capps et al., 1973). In particular the hydrogen absorption opacity is much less than the free electron opacity (see Figure 2 of Coyne and Kruszewski, 1969). No suggestion is offered to explain the variability of the polarization in $\chi$ Oph.

\section{Models}

A consistent model must explain the major observational results discussed in the previous sections and summarized here: (1) the amount of intrinsic linear 
polarization in $\mathrm{Be}$ and shell stars does not exceed $\sim 1.5 \% ;(2)$ there is a characteristic wavelength dependence, $p(\lambda)$, for this polarization which differentiates it from that for the interstellar medium. There are differences in $p(\lambda)$ from star to star. The chief characteristics of $p(\lambda)$ are a decrease in the ultraviolet polarization, a polarization maximum at $\sim 0.5 \mu \mathrm{m}$, a second maximum or, at least, a plateau longwards of the Paschen limit and a decrease towards $2 \mu \mathrm{m}$; (3) the polarization is variable with an amplitude on the average about $40 \%$ of its mean value. There are short term variations on a time scale of days superimposed on longer term variations on a time scale of years. Some stars show rapid variations over fractions of an hour; (4) shell stars show a decrease of polarization in the emission lines. In some stars the emission flux may be partially polarized. Some shell stars show peculiar polarization effects in the blue wings of the $\mathrm{H} \alpha$ emission lines.

In general one can think of producing polarization in a star or circumstellar environment by one of the following processes: (1) radiative transfer in a stellar atmosphere which gives unequal polarizations at various regions over a stellar surface so that, when non-sphericity is introduced by flattening, rotational distortion, pole-on-equator radiation differences, eclipses, etc., the integrated flux is polarized; (2) a circumstellar envelope of aligned particles which are either geometrically asymmetric or optically anisotropic; (3) a circumstellar scattering envelope whose configuration is asymmetric.

With respect to number (1) Collins (1970) and Ruciński (1970) have shown that the transfer equations developed for a plane parallel rotationally distorted non-grey atmosphere yield polarizations in the visible region of the spectrum not greater than $0.1 \%$. Cassinelli and Haisch (1974), using transfer equations developed by Cassinelli and Hummer (1971) for Rayleigh scattering in an atmosphere whose density followed an $r^{-N}$ law $(N=2,3)$, investigated the polarization produced by pure electron scattering atmospheres that are geometrically extended. Because of strong forward peaking of the radiation field polarizations as large as $50 \%$ are predicted at the limb of an extended atmosphere (compare to $11 \%$ predicted by Chandrasekhar (1946) for a plane parallel model). Polarization from the integrated flux from such an atmosphere is investigated for two geometrical shapes, a disk model and a Roche model, shown in Figure 11. Geometrically the disk model is characterized by ' $d$ ', the radius of the core centered in a disk of thickness $2 d$. It is assumed that most of the radiation comes from the core and that the material outside the core is optically thin, $\tau \gtrless 1.0$. Polarizations for the integrated flux for a core at optical depth of one are as large as $6.6 \%$, the exact value depending or the density law used. For the Roche model a maximum polarization of $\sim 2 \%$ is obtained for a core at an optical depth of about 2. For this model the core is defined as the volume within a distance of 0.4 times the polar radius where the isodensity surface is distorted by less than $1 \%$. In both of these models the direction of the net polarization is parallel to the polar axis of the star. Haisch and Cassinelli (1976) include absorptive opacities and calculate the $p(\lambda)$ for both the Roche models and the disk model. The Roche model does not yield polarizations greater than $0.3 \%$. While a sufficient amount of polarization can be provided by the disk model the observed wavelength dependence of the polarization cannot be produced.

A model geometrically similar to the disk model of Cassinelli and Haisch (1974) 
was proposed by Coyne and Kruszewski (1969). In this model, however, the star is simply a point source of flux. The polarization is not produced as a result of the transfer of radiation in the star (in particular by forward peaking of the radiation field) but simply by electron scattering of the radiation from a point source through a flattened disk of hydrogen plasma. The wavelength dependence of the polarization $p(\lambda)$ is obtained by absorption and emission processes in the hydrogen plasma which modify the otherwise neutral polarization produced by electron scattering. In other words polarized radiation is selectively absorbed and unpolarized or partially polarized flux is added.
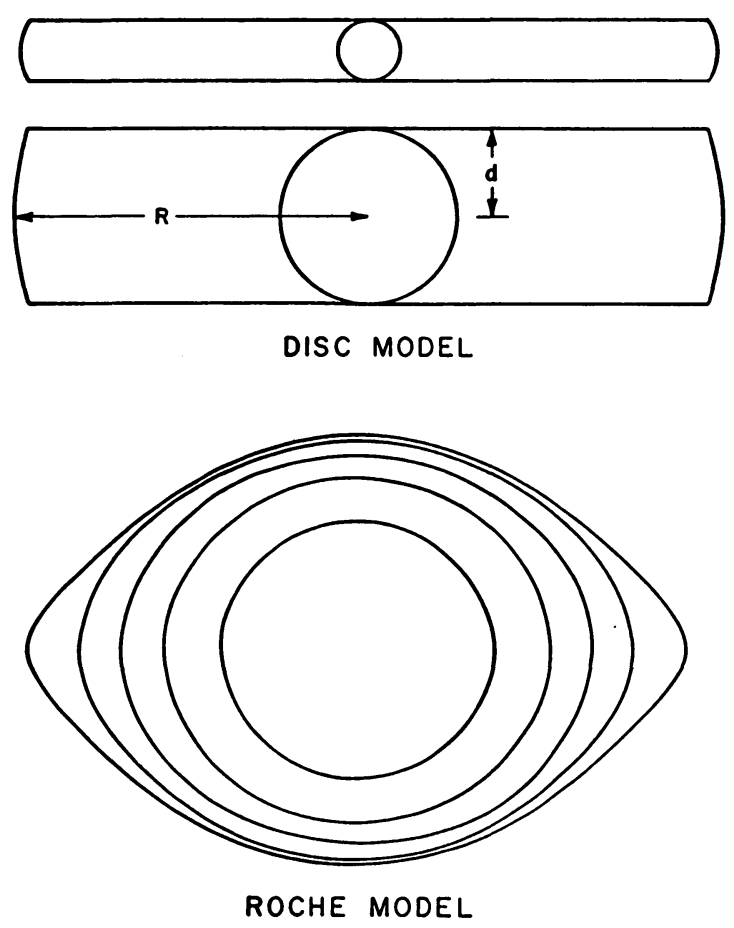

Fig. 11. Two models used by Cassinelli and Haisch (1974) and Haisch and Cassinelli (1976) to compute the polarization produced in electron scattering atmospheres that are geometrically extended.

In order to have these selective processes change the otherwise neutral polarization, it is necessary that the two sources of opacity, electron scattering and hydrogen absorption, be about equal such that the dominance of one over the other varied with wavelength. Coyne and Kruszewski (1969) showed that this was true for hydrogen plasmas with electron temperatures of $T_{e}=10000 \mathrm{~K}$ and electron densities of $N_{e}=10^{12} \mathrm{~cm}^{-3}$ (see Figure 3). With such electron densities sufficient optical depths to give the observed amounts of intrinsic polarization $(0.5$ to $1.5 \%)$ were obtained for path lengths of the order of three stellar radii. Polarization produced within this region would then be further modified by absorption and emission processes outside this region to distances of about ten stellar radii. 
These parameters of the model compare favorably with what we otherwise know of the extended envelopes about Be stars. From measured line intensities it is estimated that the radius of the emitting region is ten stellar radii and the electron density is of the order of $10^{13} \mathrm{~cm}^{-3}$ (Burbidge and Burbidge, 1956). From the width of the stellar absorption lines and the apparent speed of rotation of the emitting gas it is deduced that the main body of gas lies two or three stellar radii from the star (Huang, 1972, 1973). Kitchin (1970) from an analysis of the emission line profiles determines that the radius of the emitting region for $\zeta$ Tau is 11 stellar radii. Thus the model of an extended envelope with a more dense region extending to $\sim 3$ stellar radii and a less dense region extending to $\sim 10$ stellar radii is consistent with both the polarimetric and spectral data. The regions, of course, are not discretely separated but in the main the polarization by electron scattering is produced in the inner envelope region and is selectively modified by absorption and emission processes in the outer region. For instance, the unpolarized (or partially polarized) emission flux which explains the observed decrease in polarization in the emission lines of shell stars is produced in this outer region.

A degree of sophistication was added to this envelope model by Capps et al. (1973) who calculated the flux and polarization from 0.3 to $3 \mu \mathrm{m}$ for a range in $T_{e}$ from 8000 to $20000 \mathrm{~K}$ and for $N_{e}$ from $10^{11}$ to $10^{12} \mathrm{~cm}^{-3}$. It is shown that the wavelength dependence of the polarization is mainly due to emission in the disk. The variation of $P$ between about 0.9 and $2.2 \mu \mathrm{m}$ cannot be explained by recombination radiation alone. Free-free emission is required. The shape of the near infrared part of the $p(\lambda)$ curve depends on the relative contribution of these two processes which in turn is quite sensitive to the values of $T_{e}$ and $N_{e}$.

In Figure 12 a comparison of model calculations and polarimetric observations for $\zeta$ Tau with a group of high latitude Be stars (Coyne, 1971) is given. For $\zeta$ Tau the model fits very well. The departure of the high latitude Be stars in the red and near infrared indicates that free-free emission is a less dominant mechanism for these stars. A slight change in the model parameters would give a fit to the high latitude $\mathrm{Be}$ star curve. The same model is plotted in Figure 4 with observations of $\zeta$ Tau, $\gamma$ Cas, $48 \mathrm{Lib}$ and $\psi$ Per and in Figure 13 with observations of $\phi$ Per. Here again slight changes in $T_{e}$ and $N_{e}$ would fit the observations for the various stars by changing the relative contribution of free-free and recombination transitions. While $\phi$ Per and $\zeta$ Tau are comparable, $\gamma$ Cas has a greater and $48 \mathrm{Lib}$ a lesser contribution from free-free as compared to recombination energy.

Models which would rely upon alignment of geometrically asymmetric or optically anisotropic particles do not seem to be viable. A physical mechanism for the alignment is not available and the appropriate scattering theory has not been developed. Even in those Be stars where, from infrared measurements, one might deduce the presence of grains, the thermal flux is not polarized indicating that the grains are not aligned.

Some combination of the electron scattering models proposed by Cassinelli and Haisch (1974), Haisch and Cassinelli (1976), and Capps et al. (1973) appears to be the most fruitful approach to the problem. The variation of the polarization in the wings of emission lines will undoubtedly require selective processes in the radiative transfer calculations of Haisch and Cassinelli (1976). 


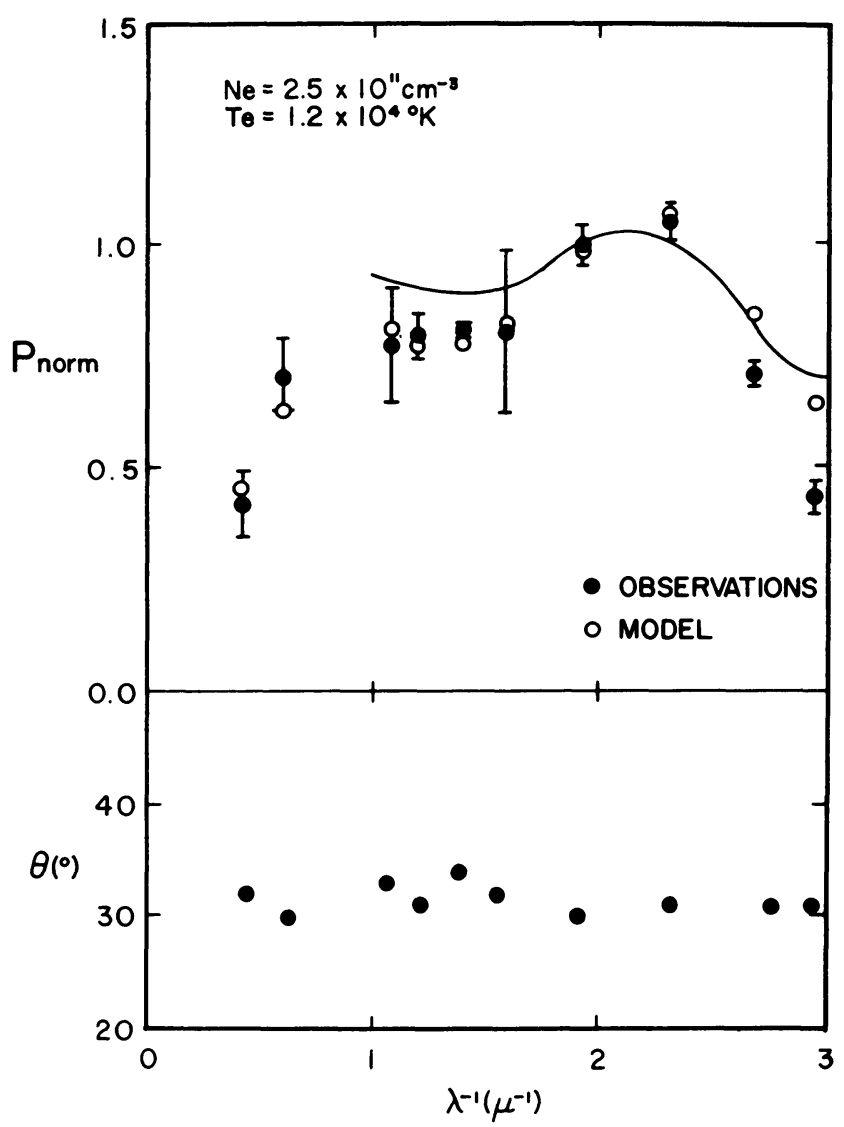

Fig. 12. Model calculations (open circles) for polarization produced in an electron scattering disk about $\zeta$ Tau are compared to observations of this star (solid circles) and observations of high galactic latitude $\mathrm{Be}$ stars (solid line).

We have not yet considered the possibility of large grains (i.e. in the Mie scattering range) in the extended envelope of Be stars as polarizing scatterers. Indeed there is some uncertainty as to whether the observed infrared fluxes in Be stars can be explained by free-free emission or whether it is necessary to evoke thermal reemission by grains of absorbed high energy flux (see Stein, 1972). The classical Be stars whose polarization we have considered here give no evidence of the presence of grains. We mention two cases of B-type stars for which there is both polarimetric and infrared evidence for the existence of Mie scattering grains. These are: HD 45677 (Coyne and Vrba, 1976) which shows a $p(\lambda)$ curve which varies on a time scale of about one-half year from a polarization continuously increasing into the near infrared to a curve with a maximum at $\sim 0.6 \mu \mathrm{m}$; and HD 44179 (Cohen et al., 1975) which shows a polarization continuously increasing into the near infrared.

It appears that the most urgent need theoretically is to evaluate the relative merits of radiative transfer processes in a stellar atmosphere and electron scattering in an extended stellar envelope. The difficulty with these exclusively photospheric models 


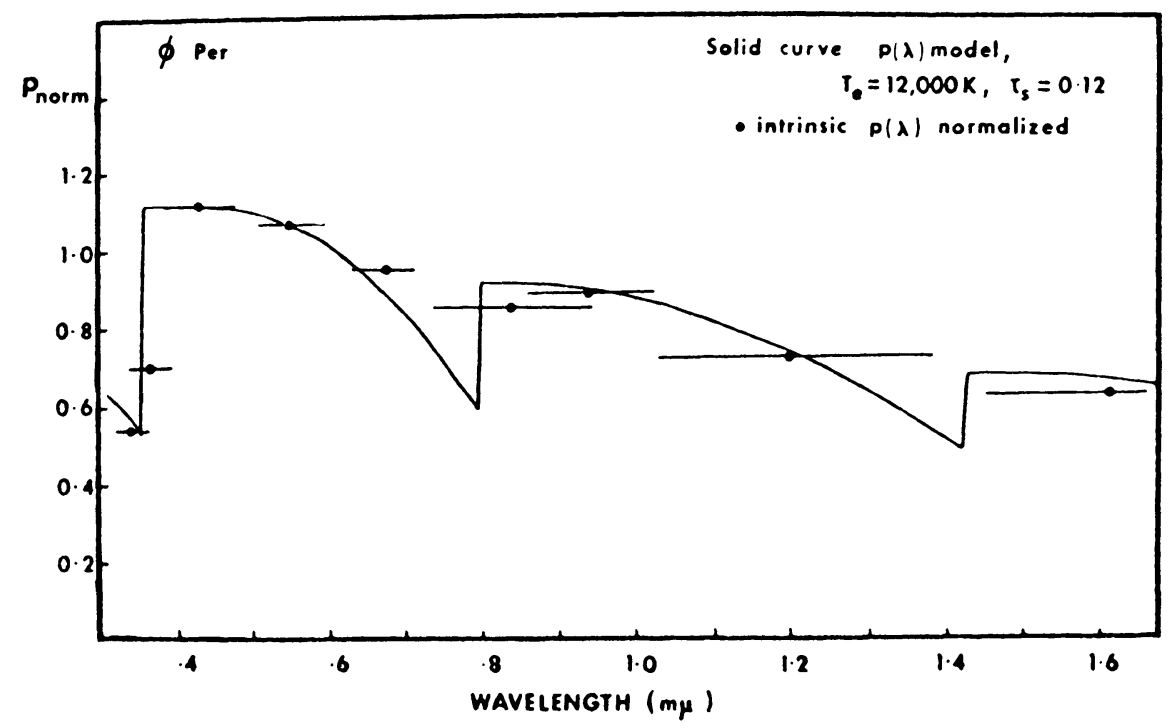

Fig. 13. Polarization observations of $\phi$ Per, after removing the effects of the interstellar polarization, are compared to model calculations of polarization produced in a flattened disk with an electron-scattering optical depth of $\tau_{s}=0.12$.

is to explain the time variability of the polarization, especially that which occurs on a short time scale of fractions of a day. Such variations could be more reasonably explained by dynamical processes occurring in an extended envelope. On the other hand the assumption in the electron-scattering extended envelope model of an unpolarized point source of radiation at the center of a disk whose height is two stellar radii with the main body of gas lying within three stellar radii and extending to ten stellar radii is only of limited validity. Undoubtedly contributions from processes occurring in both the stellar photosphere and the extended disk are necessary.

Observationally, the most urgent need is for simultaneous high resolution spectrophotometry and polarimetry across emission and absorption lines and across the hydrogen discontinuities. One could then perhaps begin to distinguish at various depths in the extended envelope the various processes of polarization and modification of the polarization. For instance one would like to make a detailed comparison of the polarization in shell lines, in the stellar lines, in emission lines, in the wings of all of these lines and in the continuum and to study the changes in the polarization with changes in the line profiles. Wide- and intermediate-band measurements between 0.6 and $2.2 \mu \mathrm{m}$ over a variety of types of $\mathrm{Be}$ and shell stars would be very valuable. The $p(\lambda)$ in this region of the spectrum is very sensitive to the relative contributions of free-free emission and recombination energy which in turn depend on the relative values of the electron temperature and density in the extended envelope. In fact this may be the most sensitive region of the spectrum for determining these parameters. Finally, it would be valuable to have a polarimetric survey of peculiar Be stars and especially of those which show abnormally large infrared excesses. A concentration on the red and infrared region of the spectrum is suggested since this is where peculiarities in the $p(\lambda)$ curve have been known to 
occur. The expectation of such a survey would be to detect the presence of grains and eventually to evaluate the relative contribution they make to the analysis of extended envelopes in Be stars.

\section{References}

Angel, J. R. P. and Landstreet, J. D.: 1970a, Astrophys. J. Letters 160, L147.

Angel, J. R. P. and Landstreet, J. D.: 1970b, Astrophys. J. Letters 162, L61.

Appenzeller, I. and Hiltner, W. A.: 1967, Astrophys. J. 149, 353.

Behr, A.: 1959, Nach. Akad. Wiss. Göttingen 2; Math-Phys. Kl., No. 7, 185 (=Veröff. Göttingen, No. 126).

Bernacca, P. D. and Perinotto, M.: 1971, Contr. Obs. Astron. Univ. Padova in Asiago, No. 250.

Boyarchuk, A. A. and Kopylov, I. M.: 1964, Crimea Izv. 32, 44.

Burbidge, G. R. and Burbidge, E. M.: 1953, Astrophys. J. 117, 407.

Burbidge, G. R. and Burbidge, E. M.: 1956, Vistas in Astronomy 2, 1446.

Capps, R. W., Coyne, G. V., and Dyck, H. M.: 1973, Astrophys. J. 184, 173.

Cassinelli, J. P. and Haisch, B. M.: 1974, Astrophys. J. 188, 101.

Cassinelli, J. P. and Hummer, D. C.: 1971, Monthly Notices Roy. Astron. Soc. 153, 9.

Chandrasekhar, S.: 1946, Astrophys. J. 103, 351.

Clarke, D. and Grainger, J. F.: 1966, Ann. Astrophys. 29, 355.

Clarke, D. and McLean, I. S.: 1974, Monthly Notices Roy. Astron. Soc. 167, 27p.

Clarke, D. and McLean, I. S.: 1975, Monthly Notices Roy. Astron. Soc. 172, 545.

Clarke, D. and McLean, I. S.: 1976, Monthly Notices Roy. Astron. Soc. 174, 335.

Clarke, D., McLean, I. S., and Wyllie, T. H. A.: 1975, Astron. Astrophys. 43, 215.

Cohen, M., Anderson, C. M., Cowley, A., Coyne, G. V., Fawley, W., Gull, T. R., Harlan, E. A., Herbig, G. H., Holden, F., Hudson, H. S., Jakoubek, R. O., Johnson, H. M., Merrill, K. M., Schiffer, F. H. III, Soifer, B. T., and Zuckerman, B.: 1975, Astrophys. J. 179, 15.

Collins, G. W.: 1970, Astrophys. J. 159, 583.

Coyne, G. V.: 1970, Astrophys. J. 161, 1011.

Coyne, G. V.: 1971, Specola Vatic. Ric. Astron. 8, 201.

Coyne, G. V.: 1974, Monthly Notices Roy. Astron. Soc. 169, 7p.

Coyne, G. V.: 1975a, Astron. Astrophys., in press.

Coyne, G. V.: 1975b, Specola Vatic. Ric. Astron. 8, 533.

Coyne, G. V. and Gehrels, T.: 1967, Astron. J. 72, 887.

Coyne, G. and Kruszewski, A.: 1969, Astron. J. 74, 528.

Coyne, G. V. and McLean, I. S.: 1975, Astron. J. 80, 702.

Coyne, G. V. and Vrba, F. J.: 1976, Astrophys. J., in press.

Coyne, G. V., Gehrels, T., and Serkowski, K.: 1974, Astron. J. 79, 581.

Derviz, T.: 1970, Astrophysics (Astrofizika) 6, 351.

Eggen, O. J., Mathewson, D. S., and Serkowski, K.: 1967, Nature 213, 1216.

Feinstein, A.: 1968, Z. Astrophys. 68, 29.

Feinstein, A.: 1976, this volume, p. 149.

Gray, D. F. and Marlborough, J. M.: 1974, Astrophys. J. Suppl. 27, 121.

Haisch, B. M. and Cassinelli, J. P.: 1976, this volume, p. 375.

Hall, J. S.: 1949, Science 109, 166.

Hall, J. S.: 1958, Publ. U.S. Naval Obs. 17, Part VI.

Hall, J.S. and Mikesell, A. H.: 1950, Publ. U.S. Naval Obs., Washington, D.C., 2nd Series, XVII, Part I.

Harrington, J. P. and Collins, G. W.: 1968, Astrophys. J. 151, 1051.

Hayes, D.: 1975, Publ. Astron. Soc. Pacific 87, 609.

Hayes, D. P. and Illing, R. M. E.: 1974, Astron. J. 79, 1430.

Hiltner, W. A.: 1949, Science 109, 165.

Hiltner, W. A.: 1956, Astrophys. J. Suppl. 2, 389.

Huang, Su-Shu: 1972, Astrophys. J. 171, 549.

Huang, Su-Shu: 1973, Astrophys. J. 183, 541.

Illing, R. M. E.: 1973, Dissertation, Physics Department, Columbia University.

Kitchin, C. R.: 1970, Monthly Notices Roy. Astron. Soc. 150, 455.

Kruszewski, A.: 1974, in T. Gehrels (ed.), Planets, Stars and Nebulae Studied with Photopolarimetry, University of Arizona Press, Tucson, p. 845. 
Lesh, J. R.: 1968, Astrophys. J. Suppl. 17, 371.

McLean, I. S.: 1974, Dissertation, The University of Glasgow, Scotland.

McLean, I. S. and Clarke, D.: 1976, this volume, p. 261.

Merrill, P. W.: 1956, Vistas in Astronomy 2, 1375.

Nagirner, D. T.: 1962, Trudy Leningrad Astron. Obs. 19, 79.

Öhman, Y.: 1934, Nature 134, 534.

Öhman, Y.: 1946, Astrophys. J. 104, 460.

Poeckert, R.: 1975a, Astrophys. J. 196, 777.

Poeckert, R.: 1975b, private communication.

Poeckert, R. and Marlborough, J. M.: 1976, this volume, p. 277.

Ruciński, S. M.: 1970, Acta Astron. $20,1$.

Schild, R. E.: 1966, Astrophys. J. 146, 142.

Schild, R. E.: 1973, Astrophys. J. 179, 221.

Sen, K. K. and Lee, W. M.: 1961, Publ. Astron. Soc. Japan 13, 263.

Serkowski, K.: 1968, Astrophys. J. 154, 115.

Serkowski, K.: 1970, Astrophys. J. 160, 1083.

Serkowski, K.: 1975, private communication.

Serkowski, K., Mathewson, D. S., and Ford, V. L.: 1975, Astrophys. J. 196, 261.

Shakhovskoi, N. M.: 1962, Astron. Circ. U.S.S.R., No. 228.

Shakhovskoi, N. M.: 1964, Astron. Zh. 41, 1042 (transl. in Soviet Astron. AJ 8, 833).

Slettebak, A. and Howard, R. F.: 1955, Astrophys. J. 121, 102.

Stein, W. A.: 1972, Publ. Astron. Soc. Pacific 84, 627.

Uesugi, A. and Fukuda, I.: 1970, Contr. Inst. Astrophys. and Kwasan Obs. Univ. of Kyoto, No. 189.

Vitrichenko, E. A. and Efimov, Y. S.: 1965, Izv. Krymsk. Astrophys. Obs. 34, 114.

Zellner, B. and Serkowski, K.: 1972, Publ. Astron. Soc. Pacific 84, 619.

\section{DISCUSSION}

Haisch: I would just like to point out that the emission feature in the Balmer lines is not just polarized because it is subsequently scattered, but the resonance scattering process itself produces polarization, which makes the problem more difficult.

McLean: I would just emphasize that the polarimetric variability observed in $\zeta$ Tau over a time interval of some $\mathbf{4 0}$ minutes was neither periodic or irregular. What was observed was a steady decrease in the degree of polarization $(p)$ recorded by a monitor channel tuned to the continuum near $\mathrm{H} \beta$. The effect was only apparent 'after the fact', when the data were reduced. A similar observation two weeks later revealed no such changes but the degree of polarization observed was smaller than the lowest value recorded on the night which revealed the variability. Strong night to night changes in $p$ have been reported by other workers. 